\title{
Short-course radiotherapy and chemotherapy for conversion surgery in patients with unresectable metastatic rectal cancer: a preliminary case series study
}

\author{
Youngbae Jeon ${ }^{1}$, Kyoung-Won Han¹, Seok Ho Lee², Sun Jin Sym³ ${ }^{3}$ Seung Joon Choi ${ }^{4}$, Seung Yeon Ha ${ }^{5}$, Jeong-Heum Baek ${ }^{1}$ \\ ${ }^{1}$ Division of Colon and Rectal Surgery, Department of Surgery, ${ }^{2}$ Department of Radiation Oncology, ${ }^{3}$ Division of Hematology and Oncology, \\ Department of Internal Medicine, Departments of ${ }^{4}$ Radiology and ${ }^{5}$ Pathology, Gil Medical Center, Gachon University College of Medicine, Incheon, \\ Korea
}

Purpose: Curative treatment is challenging in patients with locally advanced rectal cancer and unresectable metastases. The aim of this study was to evaluate the clinical outcomes of short-course radiotherapy (RT) followed by systemic chemotherapy for patients with rectal cancer with mesorectal fascia (MRF) involvement and unresectable distant metastases.

Methods: The study included consecutive patients diagnosed as having metastatic mid-to-low rectal cancer treated with short-course RT followed by systemic chemotherapy for conversion radical or palliative surgery between 2014 and 2019 at Gil Medical Center. The patients had primary rectal tumors involving the MRF and unresectable distant metastases. The treatment strategies were determined in a multidisciplinary team discussion.

Results: Seven patients (five men and two women) underwent short-course RT ( $5 \times 5 \mathrm{~Gy}$ ) and preoperative systemic chemotherapy. The median age was 68 years (range, 46-84 years), and the median distance from the anal verge to the primary tumor was 6.0 $\mathrm{cm}$ (range, $2.0-9.0$ $\mathrm{cm}$ ). During the median follow-up period of 29.4 months, three patients underwent conversion radical surgery with R0 resection, two underwent palliative surgery, and two could not undergo surgery. No postoperative major morbidity or mortality occurred. The patients who underwent conversion complete radical surgery showed good long-term survival outcomes, with an overall survival time of 29.4-48.8 months and progression-free survival time of 14.7-41.1 months.

Conclusion: Short-course RT followed by systemic chemotherapy could provide patients with unresectable stage IV rectal cancer a chance to undergo to conversion radical surgery with good long-term survival outcomes.

Keywords: Radiotherapy, Rectal cancer, Metastasis, Surgery

Received: Jun 3, 2021 Revised: Jul 28, 2021 Accepted: Oct 15, 2021

Correspondence to: Jeong-Heum Baek

Division of Colon and Rectal Surgery, Department of Surgery, Gil Medical Center, Gachon University College of Medicine, 21 Namdong-daero 774beon-gil, Namdong-gu, Incheon 21565, Korea

Tel: +82-32-460-8428, Fax: +82-32-460-3247

E-mail: gsbaek@gilhospital.com

ORCID: Youngbae Jeon (https://orcid.org/0000-0003-1322-9536), Kyoung-Won Han (https://orcid.org/0000-0001-5183-6375), Seok Ho Lee (https://orcid. org/0000-0003-0785-2831), Sun Jin Sym (https://orcid.org/0000-0002-13406291), Seung Joon Choi (https://orcid.org/0000-0003-3861-7682), Seung Yeon Ha (https://orcid.org/0000-0001-7071-1623), Jeong-Heum Baek (https://orcid. org/0000-0001-9124-8041)

*This article was presented as an e-poster at Seoul International Symposium of Surgical Oncology 2020 (SISSO 2020), Seoul, Korea, July 17-18, 2020, and wined an Outstanding Poster Award.

Copyright (C) 2021 Korean Society of Surgical Oncology

This is an Open Access article distributed under the terms of the Creative Commons Attribution Non-Commercial License (http://creativecommons.org/licenses/by-nc/4.0) which permits unrestricted non-commercial use, distribution, and reproduction in any medium, provided the original work is properly cited.

\section{INTRODUCTION}

Long-course radiotherapy (RT) with concurrent systemic chemotherapy followed by total mesorectal excision after 6-10 weeks is a standard treatment for patients with locally advanced mid-to-low rectal cancer [1]. However, no optimal treatment has been established yet for patients with initially unresectable metastatic rectal cancer [2]. In these patients, the conventional long-course concurrent chemoradiotherapy is not suitable because of the risk of progression of the metastatic lesions. The control of metastatic disease depends more on systemic chemotherapy than on external RT. Previous studies showed that unresectable metastatic lesions are reduced in size by systemic chemotherapy, enabling surgical treatment $[3,4]$. However, concerns that palliative systemic chemotherapy alone is less effective than RT for reducing the size of primary tumors have also emerged $[1,5]$. Primary tumor progression often 
leads to tumor-related complications such as bleeding, perforation, and obstruction, which may require emergency surgery and are associated with increased morbidity and mortality [6]. Therefore, both local treatment for the primary tumor and systemic treatment for the metastatic lesions are crucial to control the overall disease progression.

The management of metastatic rectal cancer has not been standardized. Thus, various treatment strategies have been attempted. Shin et al. [7] suggested that upfront chemotherapy and shortcourse RT with delayed surgery might be an alternative treatment for patients with rectal cancer with mesorectal fascia (MRF) involvement and a synchronous and potentially distant metastasis. Bisschop et al. [8] reported performing short-course RT followed by neoadjuvant systemic chemotherapy and a subsequent radical surgery for patients with stage IV rectal cancer with potentially resectable metastases. However, studies are lacking regarding treatment strategies for patients with rectal cancer with MRF involvement and unresectable distant metastases. The study reported preliminary clinical outcomes and focused on the role of short-course RT with systemic chemotherapy for conversion surgery in patients with MRF involvement of rectal cancer and unresectable metastases.

\section{METHODS}

\section{Patient selection}

We retrospectively reviewed consecutive patients with rectal cancer with MRF involvement and unresectable distant metastasis who received short-course RT and preoperative systemic chemotherapy for conversion radical or palliative surgery between 2014 and 2019 at Gachon University Gil Medical Center. Patients with hereditary rectal, recurrent rectal, and multiple primary cancers were excluded. Patients were selected by searching through the Clinical Research Data Warehouse system, and detailed data were collected from the patients' electronic medical records in Gil Medical Center. Therefore, informed consent was waived. Institutional review board approval was obtained from the ethics committee of our hospital (approval no. GCIRB2020-452).

\section{Assessment parameters and definitions}

Baseline patient characteristics including age, sex, MRF involvement, primary tumor location, metastatic sites, RT dose and duration, chemotherapy regiment and duration, and tumor stage were collected. MRF involvement was determined by preoperative magnetic resonance imaging (MRI) with reading of radiology specialists. It contained both MRF invasion and MRF threatening lesions. The resectability was determined by colorectal surgeons and radiology specialists through multidisciplinary discussion. Conversion surgery is defined as surgery with the curative aim of radical resection in initially unresectable metastatic rectal cancer after preoperative RT and chemotherapy. Palliative surgery is defined as primary tumor resection or stoma diversion to improve patient's symptoms and prevent primary tumor-related complications. Tumor regression grade (TRG) after RT was stratified according to the American Joint Committee on Cancer (AJCC) grade classification; compete regression (TRG 0), moderate response (TRG 1), minimal response (TRG 2), poor response (TRG3).

\section{Chemotherapy and RT}

The treatment strategies were determined in a multidisciplinary team discussion. The patients received short-course RT before undergoing a preoperative systemic chemotherapy (Fig. 1). All the patients received RT at $25 \mathrm{~Gy}$ in five fractions for 5 days. Within 2 weeks after completion of the RT, systemic chemotherapy was started with at least three cycles of the mFOLFOX6 (5-fluorouracil, leucovorin, oxaliplatin) or FOLFIRI (5-fluorouracil, leucovorin, irinotecan) regimen with target agents such as bevacizumab (Avastin) or cetuximab (Erbitux) according to the patients' RAS mutant status. In the patients who had already received palliative chemotherapy, preoperative chemotherapy was administered with the same regimen.

\section{Tumor staging workup and follow-up}

Tumor staging was described on the basis of the TNM staging system from the AJCC 8th edition. Digital rectal examination, abdominopelvic computed tomography, MRI, and colonoscopy were used to evaluate the rectal cancer stage. MRI was used to

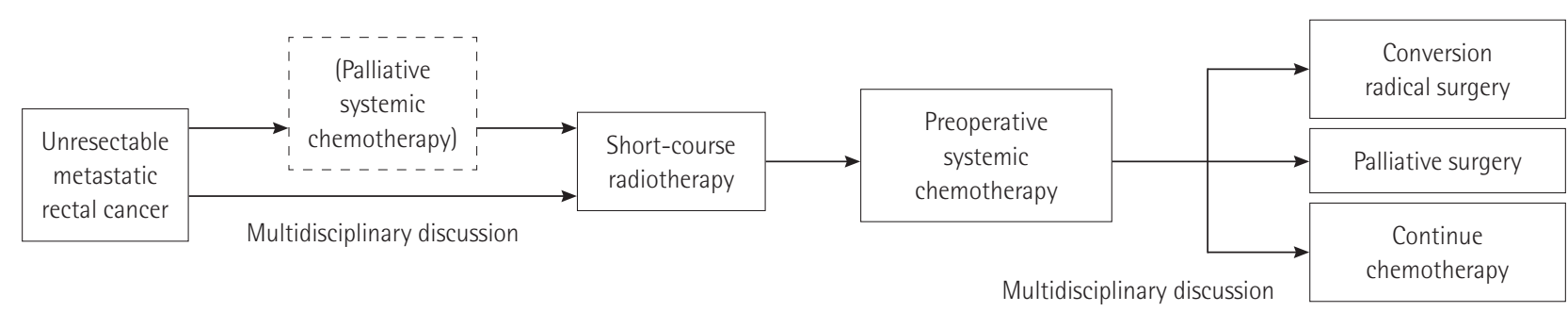

Fig. 1. Schematic diagram of the treatment protocol. 
evaluate the local extent of the tumors, infiltration of the MRF, and invasion of the surrounding lymph nodes. Patients who received short-course RT in the first week were reevaluated after every three systemic chemotherapy cycles [9]. Patients who underwent conversion surgery received additional chemotherapy every 2 weeks with a palliative chemotherapy regimen.

\section{RESULTS}

\section{Patients' characteristics and treatment}

The patients' characteristics and treatments are described in Table 1. The median age was 68 years (range, $46-84$ years). Of the patients, five were male and two were female. One case was classified as T3 with threatened MRF by the initial clinical stage, and the other six cases were classified as T4 with MRF involvement. All the cases were classified as $\mathrm{N} 2$. The median distance from the anal verge was $6 \mathrm{~cm}$ (range, 2.4-9 cm). There were four patients with liver only metastases, a patient with liver and lung metastases, a patient with liver and peritoneal metastases, and the other one patient with peritoneal metastases. In all patients, there was no early side effect such as radiation proctitis during RT periods and a month follow-up. During the median follow-up period of 29.4 months, three patients underwent conversion radical surgery with R0 resection, two underwent palliative surgery, and two could not undergo surgery. The preoperative image studies of the patients who were achieved radical surgery showed significantly decreased metastatic and primary tumor burden (Fig. 2).

\section{Clinical outcomes of conversion radical surgery}

Regarding the three patients who underwent conversion radical surgery, the interval from the last RT to the radical surgery ranged from 13.6 to 16.6 weeks. Each surgery took 540, 420, and 360 minutes, respectively. All the radical surgeries achieved R0 resection. One patient was pathologically diagnosed as having ypT4, and the other two patients were diagnosed as having ypT3 after surgery. None of the patients had significant postoperative complications and mortality. The pathologic TRG after RT was grade 2, grade 1, and grade 3, respectively. They showed an overall survival time of 29.4-48.8 months and a progression-free survival time of $14.7-$ 41.1 months. Among these patients, two patients died as cancer progression. Patient number 1 received hospice care for a month until death, and patient number 3 presented multi-organ failure and died 6 months after last chemotherapy. The other one patient was alive with a progression-free survival time of 41.1 months up to the last follow-up date. All of the three patients were initially recurred and progressed on hematogenous liver metastases. The detailed outcomes of conversion radical surgeries are demonstrated in Table 2.

\section{DISCUSSION}

This study is a preliminary report of initial experience of shortcourse RT and chemotherapy the patients with far advanced metastatic rectal cancer in our institution. Out of the seven patients who received short-course RT and systemic chemotherapy, three underwent conversion radical surgery with R0 resection, thereby

Table 1. Characteristics and preoperative treatment of patients who underwent short-course radiotherapy and chemotherapy

\begin{tabular}{|c|c|c|c|c|c|c|c|c|c|c|c|}
\hline $\begin{array}{r}\text { Case } \\
\text { no. }\end{array}$ & $\begin{array}{l}\text { Age } \\
\text { (yr) }\end{array}$ & Sex & $\begin{array}{l}\text { Initial } \\
\text { clinical } \\
\text { stage }\end{array}$ & $\begin{array}{l}\text { AV } \\
(\mathrm{cm})\end{array}$ & $\begin{array}{c}\text { MRF } \\
\text { involvement }\end{array}$ & $\begin{array}{c}\text { Distant } \\
\text { metastases }\end{array}$ & $\begin{array}{l}\text { RT dose } \\
\text { (Gy) }\end{array}$ & $\begin{array}{l}\text { Preoperative } 1 \text { st line } \\
\text { systemic chemotherapy } \\
\text { regimen }\end{array}$ & $\begin{array}{l}\text { Subsequent } \\
\text { treatment }\end{array}$ & $\begin{array}{c}\text { PFS from } \\
\text { initial RT } \\
\text { (mo) }\end{array}$ & $\begin{array}{c}\text { OS from } \\
\text { initial RT } \\
\text { (mo) }\end{array}$ \\
\hline 1 & 60 & M & CT4 N2 M1 & 5 & Involved & Liver & $5 \times 5$ & $\begin{array}{l}\text { Cetuximab+FOLFIRI } \\
\text { (3 cycles) }\end{array}$ & Radical surgery & 14.7 & 29.4 \\
\hline 2 & 65 & M & cT3 N2 M1 & 6 & Threatened & Liver & $5 \times 5$ & $\begin{array}{l}\text { Bevacizumab+FOLFIRI } \\
\text { (4 cycles) }\end{array}$ & Radical surgery & 41.1 & 48.8 \\
\hline 3 & 70 & $\mathrm{~F}$ & cT4 N2 M1 & 9 & Involved & Liver & $5 \times 5$ & $\begin{array}{l}\text { Bevacizumab+FOLFIRI } \\
\text { (6 cycles) }\end{array}$ & Radical surgery & 28.8 & 33.4 \\
\hline 4 & 74 & $\mathrm{~F}$ & CT4 N2 M1 & 8 & Involved & Peritoneum & $5 \times 5$ & $\begin{array}{l}\text { Cetuximab+FOLFIRI } \\
\text { (4 cycles) }\end{array}$ & Palliative surgery & 11.0 & 24.5 \\
\hline 5 & 80 & M & cT4 N2 M1 & 6 & Involved & Liver, lung & $5 \times 5$ & $\begin{array}{l}\text { Bevacizumab+FOLFOX } \\
\text { (1 cycle) }\end{array}$ & $\begin{array}{l}\text { Discontinuation of } \\
\text { treatment }\end{array}$ & 14.2 & 14.2 \\
\hline 6 & 43 & M & cT4 N2 M1 & 2.4 & Involved & Liver & $5 \times 5$ & $\begin{array}{l}\text { Bevacizumab+FOLFOX } \\
\text { (5 cycles) }\end{array}$ & Palliative surgery & 10.3 & 10.3 \\
\hline 7 & 58 & M & cT4 N2 M1 & 6 & Involved & $\begin{array}{l}\text { Liver, } \\
\text { peritoneum }\end{array}$ & $5 \times 5$ & $\begin{array}{l}\text { Bevacizumab+FOLFOX } \\
\text { (6 cycles) }\end{array}$ & $\begin{array}{l}\text { Continuation of } \\
\text { chemotherapy }\end{array}$ & 3.9 & 9.8 \\
\hline
\end{tabular}

$A V$, anal verge; MRF, mesorectal fascia; RT, radiotherapy; PFS, progression-free survival; OS, overall survival; $M$, male; F, female; FOLFIRI, 5-fluorouracil, leucovorin, irinotecan; FOLFOX, 5-fluorouracil, leucovorin, oxaliplatin. 

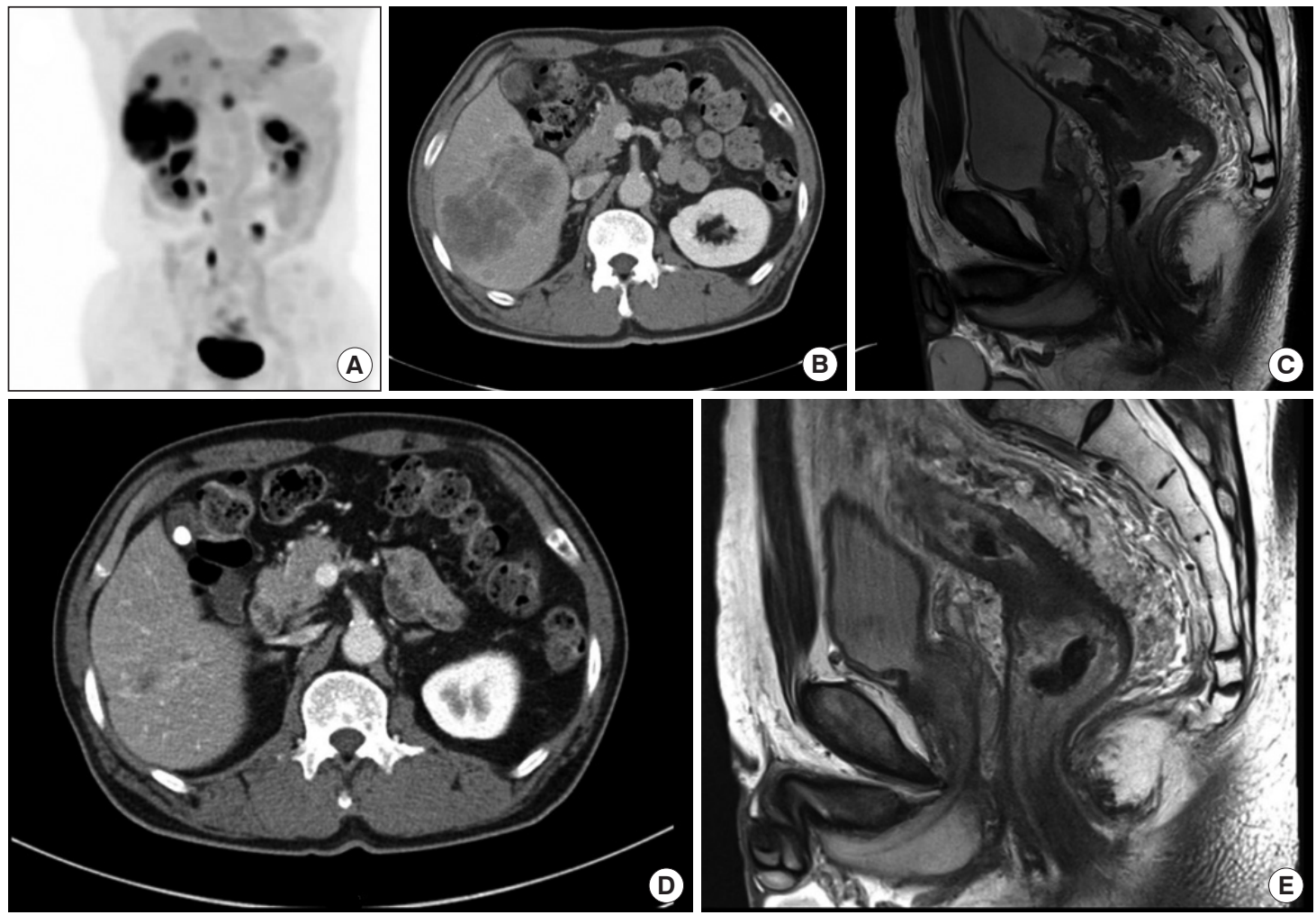

Fig. 2. Preoperative image studies of the patient who had initially unresectable hepatic metastatic rectal cancer (case no. 1). (A-C) Images before radiotherapy chemotherapy. (D, E). Images after radiotherapy and chemotherapy.

Table 2. Three cases of radical surgery after short-course radiotherapy and chemotherapy

\begin{tabular}{|c|c|c|c|c|c|c|c|c|}
\hline $\begin{array}{r}\text { Case } \\
\text { no. }\end{array}$ & $\begin{array}{l}\text { Interval from } \\
\text { the last RT to } \\
\text { surgery (wk) }\end{array}$ & Name of surgery & $\begin{array}{l}\text { AJCC tumor } \\
\text { regression } \\
\text { grade }\end{array}$ & yp stage & $\begin{array}{l}\text { Postoperative systemic } \\
\text { chemotherapy }\end{array}$ & $\begin{array}{l}\text { PFS from } \\
\text { initial RT } \\
(\mathrm{mo})\end{array}$ & $\begin{array}{l}\text { OS from } \\
\text { initial RT } \\
\text { (mo) }\end{array}$ & $\begin{array}{l}\text { Survival } \\
\text { status }\end{array}$ \\
\hline 1 & 13.6 & $\begin{array}{l}\text { Hartmann's operation, right } \\
\text { hemihepatectomy }\end{array}$ & 2 & ypT4b N2b M1 & $\begin{array}{l}\text { Cetuximab + FOLFIRI (11 cycles), } \\
\text { Bevacizumab + FOLFOX (12 cycles) }\end{array}$ & 14.7 & 29.4 & Death \\
\hline 2 & 16.6 & $\begin{array}{l}\text { Low anterior resection, } \\
\text { ileostomy diversion, right } \\
\text { hemihepatectomy }\end{array}$ & 1 & ypT3 No M1 & Bevacizumab + FOLFIRI (12 cycles) & 41.1 & 48.8 & Alive \\
\hline 3 & 13.9 & $\begin{array}{l}\text { Low anterior resection, } \\
\text { ileostomy diversion, right } \\
\text { hemihepatectomy }\end{array}$ & 3 & урT3 N1b M1 & $\begin{array}{r}\text { Bevacizumab + FOLFIRI (19 cycles), } \\
\text { Bevacizumab + FOLFOX (7 cycles) }\end{array}$ & 28.8 & 33.4 & Death \\
\hline
\end{tabular}

RT, radiotherapy; AUCC, American Joint Committee on Cancer; PFS, progression-free survival; OS, overall survival; FOLFIRI, 5-fluorouracil, leucovorin, irinotecan; FOLFOX, 5-fluorouracil, leucovorin, oxaliplatin.

good long-term survival outcomes were attained, with an overall survival time of 29.4-48.8 months and progression-free survival time of 14.7-41.1 months. Considering that patients with unresectable metastatic rectal cancer is challenging to treat and have a poor prognosis, our results are quite encouraging.

Approximately $20 \%$ of patients with rectal cancer are diagnosed as having distant metastatic disease at the time of first visit [10], of whom 75\%-90\% have unresectable metastases [11]. The prognosis of the patients is usually dismal, and the reported life expectancy was $<8-12$ months when only fluorouracil and leucovorin were administered $[12,13]$. Although the development of monoclonal antibodies such as cetuximab and bevacizumab has led to enhancement of the overall survival of patients with metastatic colorectal cancer for up to 17.9-29.9 months [14-16], if radical surgery is possible, surgical treatment is the best option to improve survival outcomes. Complete resection of hepatic metastases in patients with colorectal cancer was reported to attain a median overall survival of 30-50 months and a 5-year overall survival rate 
of up to 50\% [17]. Even though concomitant liver and lung metastases were present, complete synchronous radical resection showed significantly better overall survival outcomes in selected patients $(\mathrm{P}<0.001)$ [18]. According to Nozawa et al. [19], in patients with an initially unresectable metastatic colorectal cancer, successful conversion to radical resection after systemic chemotherapy showed long-term overall survival outcomes similar to those in patients with an initially resectable stage IV colorectal cancer who first underwent a curative surgery (3-year overall survival rate: $66 \%$ vs. $77 \%, P=0.22$ ). Therefore, conversion radical surgery could provide a survival benefit to patients with unresectable metastatic colorectal cancer.

In patients with rectal cancer with unresectable metastatic disease, the primary tumor is often far advanced with MRF involvement, which makes a surgical approach challenging. When the primary tumor is a mid-to-low rectal cancer, another intensive treatment can be chosen, which is application of an external radiation beam to the pelvic cavity to control the progression of the primary tumor. The standard long-course chemoradiotherapy leads to reduced locoregional disease, with local recurrence rates of approximately $5 \%$ [20]. However, the role of fluorouracil or capecitabine with conventional chemoradiotherapy in systemic control is inadequate because they mainly act as a radiosensitizer to improve the effect of radiation [21]. To overcome the problem regarding the control of locoregional disease and distant metastases, the Dutch phase II clinical trial suggested a treatment strategy that includes preoperative short-course RT $(5 \times 5 \mathrm{~Gy})$ followed by capecitabine and oxaliplatin with bevacizumab and a subsequent radical surgery for patients with rectal cancer with resectable or abatable metastases in the liver or lung [22]. In the follow-up study, good longterm survival outcomes were achieved, with a reported median overall survival of 3.8 years (range, $0.5-9.4$ years) and $32 \%$ of the patients remaining alive within a median follow-up period of 8.1 years [8]. However, the Dutch trial was different from the planned radical surgery for patients with potentially resectable metastases, but our study was deliberately focused on radical surgery for patients with unresectable metastases. This implies that the patients were at a more dismal stage in our study.

This research aimed to convert to radical surgery as the primary goal. If this is not possible, the secondary goal was to protect against upcoming tumor-relating complications by performing palliative primary tumor resection. Although the effectiveness of primary tumor resection is controversial, several studies reported that primary tumor resection could improve tumor-related complications and overall survival [6,23-25]. In our study, three of seven patients underwent complete resection, but the remaining four patients did not achieve surgical conversion. In the remaining four patients, we expected to perform palliative primary tumor resection, but none of them could undergo a primary tumor resection. Two of the patients underwent palliative colostomy because they had an obstruction and peritoneal seeding with frozen pelvis; thus, even a primary tumor resection was impossible. Another patient refused to receive surgery, and the remaining patient did not show tumor regression and resectability of the primary tumor in the preoperative imaging study after short-course RT. Consequently, we could not achieve the secondary goal of primary tumor resection, probably owing to several unknown reasons such as that the disease status was too far advanced to reduce the tumor burden by chemoradiotherapy in patients who failed to achieve tumor regression.

In this case series, we tried to convert to complete surgical treatment for the patients with unresectable metastatic rectal cancer treated with short-course RT and preoperative systemic chemotherapy. However, the optimal timing and durations of the RT and chemotherapy were not refined. Furthermore, the small number of cases and retrospective design are the main limitations of our study. Nevertheless, the fact that three of seven patients achieved conversion radical surgery and had good long-term survival outcomes is encouraging.

In conclusion, we found encouraging preliminary outcomes; thus, short-course RT followed by systemic chemotherapy could provide a chance to convert to radical surgery in patients with initially unresectable stage IV mid-to-low rectal cancer. More-detailed patient selection criteria and large-scale prospective randomized trials are needed to determine the absolute benefits of the present study.

\section{CONFLICT OF INTEREST}

No potential conflict of interest relevant to this article was reported.

\section{REFERENCES}

1. Sauer R, Becker H, Hohenberger W, Rodel C, Wittekind C, Fietkau $\mathrm{R}$, et al. Preoperative versus postoperative chemoradiotherapy for rectal cancer. N Engl J Med 2004;351:1731-40.

2. Adam R, Delvart V, Pascal G, Valeanu A, Castaing D, Azoulay D, et al. Rescue surgery for unresectable colorectal liver metastases downstaged by chemotherapy: a model to predict long-term survival. Ann Surg 2004;240:644-57.

3. Lam VW, Spiro C, Laurence JM, Johnston E, Hollands MJ, Pleass $\mathrm{HC}$, et al. A systematic review of clinical response and survival outcomes of downsizing systemic chemotherapy and rescue liver surgery in patients with initially unresectable colorectal liver metasta- 
ses. Ann Surg Oncol 2012;19:1292-301.

4. Devaud N, Kanji ZS, Dhani N, Grant RC, Shoushtari H, Serrano $\mathrm{PE}$, et al. Liver resection after chemotherapy and tumour downsizing in patients with initially unresectable colorectal cancer liver metastases. HPB (Oxford) 2014;16:475-80.

5. Hofheinz RD, Wenz F, Post S, Matzdorff A, Laechelt S, Hartmann JT, et al. Chemoradiotherapy with capecitabine versus fluorouracil for locally advanced rectal cancer: a randomised, multicentre, non-inferiority, phase 3 trial. Lancet Oncol 2012;13:579-88.

6. Shida D, Hamaguchi T, Ochiai H, Tsukamoto S, Takashima A, Boku N, et al. Prognostic impact of palliative primary tumor resection for unresectable stage 4 colorectal cancer: using a propensity score analysis. Ann Surg Oncol 2016;23:3602-8.

7. Shin SJ, Yoon HI, Kim NK, Lee KY, Min BS, Ahn JB, et al. Upfront systemic chemotherapy and preoperative short-course radiotherapy with delayed surgery for locally advanced rectal cancer with distant metastases. Radiat Oncol 2011;6:99.

8. Bisschop C, van Dijk TH, Beukema JC, Jansen R, Gelderblom H, de Jong KP, et al. Short-course radiotherapy followed by neoadjuvant bevacizumab, capecitabine, and oxaliplatin and subsequent radical treatment in primary stage IV rectal cancer: long-term results of a phase II study. Ann Surg Oncol 2017;24:2632-8.

9. Glynne-Jones R, Wyrwicz L, Tiret E, Brown G, Rodel C, Cervantes A, et al. Rectal cancer: ESMO Clinical Practice Guidelines for diagnosis, treatment and follow-up. Ann Oncol 2017;28(suppl_4):iv 22-40.

10. Leporrier J, Maurel J, Chiche L, Bara S, Segol P, Launoy G. A population-based study of the incidence, management and prognosis of hepatic metastases from colorectal cancer. Br J Surg 2006;93:46574.

11. Park JH, Kim TY, Lee KH, Han SW, Oh DY, Im SA, et al. The beneficial effect of palliative resection in metastatic colorectal cancer. $\mathrm{Br}$ J Cancer 2013;108:1425-31.

12. Lemmens VE, de Haan N, Rutten HJ, Martijn H, Loosveld OJ, Roumen RM, et al. Improvements in population-based survival of patients presenting with metastatic rectal cancer in the south of the Netherlands, 1992-2008. Clin Exp Metastasis 2011;28:283-90.

13. Meta-analysis Group In Cancer, Piedbois P, Rougier P, Buyse M, Pignon J, Ryan L, et al. Efficacy of intravenous continuous infusion of fluorouracil compared with bolus administration in advanced colorectal cancer. J Clin Oncol 1998;16:301-8.

14. Van Cutsem E, Kohne CH, Lang I, Folprecht G, Nowacki MP, Cascinu S, et al. Cetuximab plus irinotecan, fluorouracil, and leucovorin as first-line treatment for metastatic colorectal cancer: updated analysis of overall survival according to tumor KRAS and BRAF mutation status. J Clin Oncol 2011;29:2011-9.
15. Kabbinavar FF, Hambleton J, Mass RD, Hurwitz HI, Bergsland E, Sarkar S. Combined analysis of efficacy: the addition of bevacizumab to fluorouracil/leucovorin improves survival for patients with metastatic colorectal cancer. J Clin Oncol 2005;23:3706-12.

16. Heinemann V, von Weikersthal LF, Decker T, Kiani A, Vehling-Kaiser U, Al-Batran SE, et al. FOLFIRI plus cetuximab versus FOLFIRI plus bevacizumab as first-line treatment for patients with metastatic colorectal cancer (FIRE-3): a randomised, open-label, phase 3 trial. Lancet Oncol 2014;15:1065-75.

17. Ito K, Govindarajan A, Ito H, Fong Y. Surgical treatment of hepatic colorectal metastasis: evolving role in the setting of improving systemic therapies and ablative treatments in the 21st century. Cancer J 2010;16:103-10.

18. Lee SH, Kim SH, Lim JH, Kim SH, Lee JG, Kim DJ, et al. Aggressive surgical resection for concomitant liver and lung metastasis in colorectal cancer. Korean J Hepatobiliary Pancreat Surg 2016;20: 110-5.

19. Nozawa H, Ishihara S, Kawai K, Hata K, Kiyomatsu T, Tanaka T, et al. Conversion to resection in patients receiving systemic chemotherapy for unresectable and/or metastatic colorectal cancer-predictive factors and prognosis. Clin Colorectal Cancer 2018;17:e917.

20. Braendengen M, Tveit KM, Berglund A, Birkemeyer E, Frykholm G, Pahlman L, et al. Randomized phase III study comparing preoperative radiotherapy with chemoradiotherapy in nonresectable rectal cancer. J Clin Oncol 2008;26:3687-94.

21. Ceelen WP, Van Nieuwenhove Y, Fierens K. Preoperative chemoradiation versus radiation alone for stage II and III resectable rectal cancer. Cochrane Database Syst Rev 2009;(1):CD006041.

22. van Dijk TH, Tamas K, Beukema JC, Beets GL, Gelderblom AJ, de Jong KP, et al. Evaluation of short-course radiotherapy followed by neoadjuvant bevacizumab, capecitabine, and oxaliplatin and subsequent radical surgical treatment in primary stage IV rectal cancer. Ann Oncol 2013;24:1762-9.

23. Costi R, Mazzeo A, Di Mauro D, Veronesi L, Sansebastiano G, Violi V, et al. Palliative resection of colorectal cancer: does it prolong survival? Ann Surg Oncol 2007;14:2567-76.

24. Gulack BC, Nussbaum DP, Keenan JE, Ganapathi AM, Sun Z, Worni M, et al. Surgical resection of the primary tumor in stage IV colorectal cancer without metastasectomy is associated with improved overall survival compared with chemotherapy/radiation therapy alone. Dis Colon Rectum 2016;59:299-305.

25. Maroney S, de Paz CC, Reeves ME, Garberoglio C, Raskin E, Senthil $\mathrm{M}$, et al. Benefit of surgical resection of the primary tumor in patients undergoing chemotherapy for stage IV colorectal cancer with unresected metastasis. J Gastrointest Surg 2018;22:460-6. 